\title{
植被含水量高光谱遥感监测研究进展
}

\author{
张 峰 ${ }^{1,2}$ 周广胜 $1,2^{*}$
}

${ }^{1}$ 中国气象科学研究院, 北京 $100081{ }^{2}$ 中国科学院植物研究所植被与环境变化国家重点实验室, 北京 100093

摘 要 植被含水量是陆地植被重要的生物物理特征, 其定量遥感反演有助于植被干旱胁迫的实时监测与诊断评估。该文系 统综述了国内外利用高光谱遥感评估植被水分状况的 4 个常见植被水分指标——冠层含水量、叶片等量水厚度、活体可燃物 湿度和相对含水量的概念及其遥感估算方法研究进展, 评述了植被含水量高光谱遥感估算各类方法的优缺点, 探讨了植被含 水量高光谱遥感估算目前存在的问题, 并提出进一步的研究任务, 即服务于植被干旱胁迫的高光谱遥感监测、预警与评估。 关键词 冠层含水量; 高光谱遥感; 叶片等量水厚度; 活体可燃物湿度; 植被水分状况

张峰, 周广胜 (2018). 植被含水量高光谱遥感监测研究进展. 植物生态学报, 42, 517-525. DOI: 10.17521/cjpe.2017.0313

\section{Research progress on monitoring vegetation water content by using hyperspectral remote sensing}

ZHANG Feng ${ }^{1,2}$ and ZHOU Guang-Sheng ${ }^{1,2^{*}}$

${ }^{1}$ Chinese Academy of Meteorological Sciences, Beijing 100081, China; and ${ }^{2}$ State Key Laboratory of Vegetation and Environmental Change, Institute of Botany, Chinese Academy of Sciences, Beijing 100093, China

\section{Abstract}

Aims Vegetation water content is an important biophysical property of terrestrial vegetation, and its remote estimation can be utilized for real-time monitoring of vegetation drought stress. This paper reviewed and summarized the conception and research progress of four commonly used vegetation water indicators: canopy water content, leaf equivalent water thickness, live fuel moisture content, and relative water content. The advantage and disadvantage of various research methods were evaluated by estimating vegetation water content and identifying the limitation in monitoring vegetation water content using optical hyperspectral remote sensing techniques. Finally, the future research tasks were discussed to address issues on accurate monitoring, early warning and evaluation of vegetation drought stress.

Key words canopy water content; hyperspectral remote sensing; leaf equivalent water thickness; live fuel moisture content; vegetation water status

Zhang F, Zhou GS (2018). Research progress on monitoring vegetation water content by using hyperspectral remote sensing. Chinese Journal of Plant Ecology, 42, 517-525. DOI: 10.17521/cjpe.2017.0313

植被是陆地生态系统的重要组成部分, 植被冠 层中水分含量为 $40 \%-80 \%$ (沈艳等, 2005)。植被含 水量(vegetation water content, VWC)是植被干旱胁 迫状态的重要指示指标, 常见植被水分指标有冠层 含水量(canopy water content, CWC) (Danson et al., 1992; Clevers et al., 2008)、叶片等量水厚度 (equivalent water thickness, EWT) (Hunt Jr \& Rock, 1989; Jacquemoud \& Baret, 1990)、活体可燃物湿度 (live fuel moisture content, LFMC) (Bowyer \& Danson, 2004) 以及相对含水量(relative water content, RWC) (Hunt Jr et al., 1987; Inoue et al., 1993)。植物
水分是影响绿色植物光合作用和生物量的主要因素, 许多关键生物地球化学循环过程, 包括光合作用、 蒸发散和净初级生产力均直接与其密切相关 (Running \& Gower, 1991; Running \& Nemani, 1991; de Jong et al., 2014)。植物水分在植被功能、植被与 大气之间的水分交换和能量传输, 干旱以及火险评 估中发挥着重要作用(Peñuelas et al., 1993, 1996; Mirzaie et al., 2014), 其深入研究对于准确监测与诊 断植被环境胁迫程度(Ustin et al., 2004)、自然火潜 在发生(Chuvieco et al., 2002)以及土壤水分有效获 取(Yilmaz et al., 2008)等具有重要研究意义。 
遥感技术是一种快捷、非破坏、多尺度探测植 被生物物理(Cohen et al., 2003; Cho et al., 2007; Darvishzadeh et al., 2008a, 2008b; 任鸿瑞等, 2012; Zhang et al., 2014, 2015; Zhang \& Zhou, 2017)与生 物化学(Curran et al., 2001; Darvishzadeh et al., 2008b; 张峰和周广胜, 2014)特征的重要研究手段。 近年来, 高光谱遥感技术相对于传统的宽波段遥感, 光谱分辨率大大提高, 能够详细记录各波段反射率 值, 有效提高了植被含水量遥感反演精度, 广泛应 用于作物干旱、森林和草原火灾、土地覆盖变化以 及农作物产量监测(Zhang et al., 2010)。

当前, 植被含水量高光谱遥感估算研究在国内 外备受关注, 其准确定量遥感反演不仅对于农林和 水文研究具有重要意义, 而且对于植被干旱评估具 有不可忽视的作用(邓兵等, 2016)。植被含水量准确 定量遥感估算有助于: (1)检测植被生理状态, 水分 胁迫对植物长势和产量的影响比任何其他胁迫都要 大得多, 准确地监测或诊断叶片/冠层含水量可以 及时反映植物生理状况 (Kramer, 1983; Peñuelas et al., 1993; 张佳华等, 2007; Yi et al., 2013); (2)植被 干旱胁迫程度的实时监测与准确评估(袁文平和周 广胜, 2004; 李柏贞和周广胜, 2014; Mirzaie et al., 2014; Cao et al., 2015; Zhang \& Zhou, 2015); (3)预测 森林、草原火险发生, 而且植被含水量指标也是燃 烧效率和火险预测的重要参数(Maki et al., 2004); (4)农业灌溉决策与产量评估 (唐延林和黄敬峰, 2001; 张佳华等, 2010; Yi et al., 2013, 2014)。因此, 为更好地开展植被干旱胁迫遥感监测的方法与诊断 模型研究, 本文将综述国内外广泛使用的各类植被 水分指标及其遥感估算方法, 评述植被水分高光谱 遥感估算各类方法的优缺点, 并探讨目前存在的问 题以及未来拟开展的研究发展方向, 以期为我国植 被干旱胁迫的高光谱遥感监测、预警与评估提供重 要的科学依据。

\section{1 植被含水量度量的 4 个常见概念}

\section{1 冠层含水量}

冠层含水量 $\left(C W C, \mathrm{~g} \cdot \mathrm{m}^{-2}\right)$ 是指单位地表面积植 被冠层的含水量(Ceccato et al., 2002), 往往通过单 位叶面积水分含量 $(E W T)$ 与叶面积指数 $(L A I)$ 的乘积, 或者由叶片生物量鲜质量 $\left(m_{\mathrm{fresh}}, \mathrm{g} \cdot \mathrm{m}^{-2}\right)$ 与生物量干 质量 $\left(m_{\text {dry }}, \mathrm{g} \cdot \mathrm{m}^{-2}\right)$ 的差值进行计算(Danson et al.,
1992; Clevers et al., 2008):

$$
C W C=m_{\text {fresh }}-m_{\text {dry }}=E W T \times L A I
$$

\section{2 叶片等量水厚度}

叶片等量水厚度 $\left(E W T, \mathrm{~g} \cdot \mathrm{cm}^{-2}\right)$ 通常是指叶片尺 度单位叶面积水分含量, 也可以在冠层尺度进行计 算(Hunt Jr \& Rock, 1989; Jacquemoud \& Baret, 1990; Danson et al., 1992; Jacquemoud et al., 1996), 如公 式(2)。

$$
E W T=C W C / L A I=\left(m_{\text {fresh }}-m_{\text {dry }}\right) / L A I
$$

\section{3 活体可燃物湿度}

活体可燃物湿度 (LFMC, \%) 是指活植物体中叶 片水分与干物质的比例, 它是决定火险和火行为模 拟的重要可燃物特性(Burgan, 1996)。通过公式(3) (Yebra et al., 2013)计算:

$$
L F M C=\left(m_{\text {fresh }}-m_{\text {dry }}\right) / m_{\text {dry }}=E W T / D M C
$$

其中, 干物质含量 $\left(D M C, \mathrm{~g} \cdot \mathrm{cm}^{-2}\right)$ 是叶片 $m_{\mathrm{dry}}$ 与 $L A I$ 之 比, 即: $D M C=m_{\mathrm{dry}} / L A I$ 。

\section{4 相对含水量}

相对含水量 $(R W C, \%)$, 也称为相对膨胀度 $(R T, \%)$ (Barrs \& Weatherley, 1962; Inoue et al., 1993), 用来 测定叶片水分亏缺程度与评估植物水分状况, 它是 叶片测量时刻含水量与叶片完全膨胀饱和时刻含水 量之比(公式4)。其中, 除了叶片的 $m_{\text {fresh }}$, 还有叶片 膨胀鲜质量 $\left(m_{\text {turgid }}, \mathrm{g} \cdot \mathrm{m}^{-2}\right)$ 和 $m_{\text {dry }}$ 。

$$
R W C(\text { 或 } R T)=\left(m_{\text {fresh }}-m_{\text {dry }}\right) /\left(m_{\text {turgid }}-m_{\text {dry }}\right) \times 100
$$

\section{5 植被含水量度量指标概念之间的关系}

植被水分指标的决定因素及其相互关系如图1

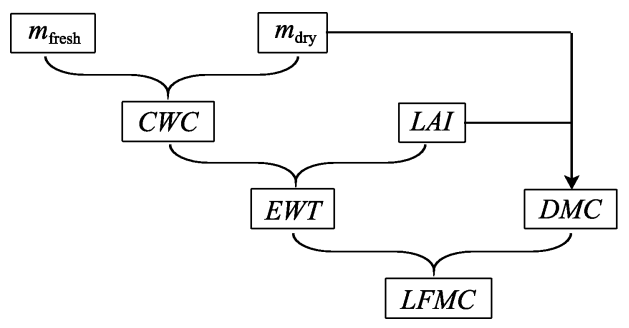

图1 植被含水量指标冠层含水量 $\left(C W C, \mathrm{~g} \cdot \mathrm{m}^{-2}\right)$ 、叶片等量 水厚度 $\left(E W T, \mathrm{~g} \cdot \mathrm{cm}^{-2}\right)$ 和活体可燃物湿度 $\left(L F M C, \mathrm{~g} \cdot \mathrm{cm}^{-2}\right)$ 之 间的关系框图。DMC, 干物质含量 $\left(\mathrm{g} \cdot \mathrm{cm}^{-2}\right)$; $L A I$, 叶面积指 数; $m_{\mathrm{dry}}$, 生物量干质量 $\left(\mathrm{g} \cdot \mathrm{m}^{-2}\right) ; m_{\mathrm{fresh}}$, 生物量鲜质量 $\left(\mathrm{g} \cdot \mathrm{m}^{-2}\right)$ 。 Fig. 1 A diagram showing the relationship of vegetation water content indicators, canopy water content $\left(C W C, \mathrm{~g} \cdot \mathrm{m}^{-2}\right)$, leaf equivalent water thickness $\left(E W T, \mathrm{~g} \cdot \mathrm{cm}^{-2}\right)$ and live fuel moisture content $\left(L F M C, \mathrm{~g} \cdot \mathrm{cm}^{-2}\right)$. DMC, dry matter content $\left(\mathrm{g} \cdot \mathrm{cm}^{-2}\right)$; $L A I$, leaf area index; $m_{\text {dry }}$, dry mass $\left(\mathrm{g} \cdot \mathrm{m}^{-2}\right) ; m_{\text {fresh }}$, fresh mass $\left(\mathrm{g} \cdot \mathrm{m}^{-2}\right)$. 
所示, $C W C$ 来源于叶片 $m_{\mathrm{fresh}}$ 和 $m_{\mathrm{dry}}$ 的差值, 而 $E W T$ 是 $C W C$ 与 $L A I$ 的比值, 进而 $E W T$ 和 $D M C$ 共同决定 $L F M C$ 的变异。植被水分指示指标中, 只有 $E W T$ 直接 与水分吸收的辐射密切相关(Yebra et al., 2013)。

\section{2 植被含水量监测指标研究进展}

围绕植被含水量高光谱遥感反演, 国内外已开 展大量研究, 然而就植被水分状况指示指标本身而 言, 目前尚缺乏统一定论。Tanner (1963)曾提出以冠 层温度来指示植物水分亏缺, 冠层温度也一度成为 诊断植被水分状况的重要手段。然而由于温度的时 空变化受环境状况的影响较大, 进而限制了该方法 的应用(王纪华等, 2000)。随后, Kriedemamn和Barrs (1983) 提出植物叶片水分状况直接影响植物的生长 发育, 而土壤水分只是间接影响因素的这一观点, 与此同时, Kramer (1983)指出植物水分状况只能从 植物本身的测定中得到。

当前, 对于植物本身可以测定的用来指示植物 水分状况的常见指标主要有: 气孔导度(Zhou et al., 2013)、叶水势(Dzikiti et al., 2010; Zhou et al., 2013)、 冠层含水量(CWC) (Danson et al., 1992; Ceccato et al., 2002; Clevers et al., 2008, 2010; Zhang \& Zhou, 2015)、叶片等量水厚度(EWT) (Danson et al., 1992; Jacquemoud et al., 1996)、活体可燃物湿度(LFMC) (Burgan, 1996; Yi et al., 2014)以及相对含水量(RWC) (Hunt Jr et al., 1987; Inoue et al., 1993; Maki et al., 2004)。叶片含水量被广泛地认为是干旱胁迫的有效 指示指标(Dzikiti et al., 2010; de Jong et al., 2012)。 关于叶水势, 研究表明与午间叶水势相比, 午间茎 水势是更好的干旱监测指标, 即午间叶水势大的变 异性并不意味着它是干旱的有效监测指标(Dzikiti et al., 2010)。LFMC不仅受叶片湿度状态的影响, 而 且与干物质的季节变化有关, 代表了供燃烧的可燃 物量的大小, 它是火险评估中用来指示植被水分状 态的最好指标(Maki et al., 2004), 但研究认为遥感 信息对于 LFMC的反演能力远不及EWT (Yi et al., 2013, 2014)。RWC 是叶片含水量与叶片完全膨胀时 最大含水量之比, 常被用来评估植物的水分状况, 人们已经利用光谱信息进行该指标的遥感估算 $(\mathrm{Qi}$ et al., 2014)。EWT指示单位叶面积的含水量, 更多 地与能量吸收有关, 其快速降低被认为是干旱胁迫 的早期指示指标(de Jong et al., 2012)。此外, CWC广
泛应用于植被水分状况的遥感监测, 它不仅与植被 水分状况有关, 而且与植被的生长发育状况密切相 关(Zhang \& Zhou, 2015), CWC的遥感反演并不需要 区分 $L A I$ 和水分信息对光谱反射率的贡献(Bowyer \& Danson, 2004; Colombo et al., 2008)。因此, 高光 谱遥感技术可实现对植被水分状况和植被干旱胁迫 的实时监测, 但是究竟哪一个植被水分指标更适合 于植被水分胁迫过程的遥感监测还有待进一步深入 研究。

\section{3 植被含水量高光谱遥感监测方法研究进展}

基于高光谱遥感信息, 多种研究方法被用来估 算植被含水量, 主要有经验方法或物理模型。早期 研究主要是利用数理统计方法建立叶片/冠层反射 率数据与叶片水分含量指标之间的关系。后来引入 物理模型, 不仅在理论上探讨植被水分与植被光谱 特征之间的内在联系, 而且方法上从单纯的统计关 系建立发展到考虑光在植被冠层中的辐射传输过程, 将光谱的遥感物理机制与植物生理过程统一起来, 建立了基于机理分析的遥感监测模型(张佳华等, 2007)。

\section{1 单波段光谱反射率法}

高光谱遥感能够监测植物水分, 其理论基础在 于植物水分能够吸收近红外(750-1300 nm) 和短波 近红外(1 300-2 $500 \mathrm{~nm}$ )区域的辐射能(Colombo et al., 2008; Yi et al., 2013, 2014)。众多研究者提出植 物在970、1200、1 450 和 $1950 \mathrm{~nm}$ 附近光谱反射率 曲线的吸收峰特征可以用来指示植物水分状况 (Rollin \& Milton, 1998; 刘畅等, 2016)。Jackson (1985)研究了棉花(Gossypium hirsutum)水分胁迫的 光谱响应, 指出作物水分胁迫能够在光谱反射率数 据中体现。Peñuelas等(1993, 1996)研究发现在近红 外波段950-970 nm范围内对应一个弱的水吸收峰, 并证明该吸收峰可以用来监测植物水分含量, 同时, 也提出了利用水分指数 $R_{970} / R_{900}(W I$ 监测小麦 (Triticum aestivum 'Soissons') 水分状况, 水分指数 可以较为灵活地反映叶片水分相对含水量、叶片水 势、气孔电导性和细胞壁弹性。研究表明, 光谱反 射率可用于诊断小麦叶片水分状况(田庆久等, 2000; 王纪华等, 2000，2001), 以及光谱 $1374-$ $1534 \mathrm{~nm}$ 范围是表征植物含水率大小的特征波段 (赵钊等, 2010)。 
此外, Danson等(1992)研究表明与水分吸收特 征相关的光谱反射率一阶微分值与叶片水分含量的 相关关系与反射率相比更显著。Michio和Tsuyoshi (1989)研究表明960 $\mathrm{nm}$ 处的导数光谱可用来监测水 稻(Oryza sativa)水分亏缺状况。Clevers等(2008)利用 970 和1 $200 \mathrm{~nm}$ 水分吸收特征的光谱一阶微分开展 CWC估算研究, 与此同时, Rollin和Milton (1998)发 现这两个水分吸收区的左斜边(1 $156 \mathrm{~nm}$ 附近)与 CWC有着较好的相关关系。Clevers等(2008)研究表 明 $970 \mathrm{~nm}$ 和 $1200 \mathrm{~nm}$ 斜率的一阶微分值对 CWC有着 较好的预测潜力, 并且 Clevers等(2010)也检验了 $970 \mathrm{~nm}$ 右斜边(1 015-1 050 nm) 一阶微分值对 CWC 的反演能力。研究证实, 光谱反射率或一阶微分反 射率的任意波段组合篮选的最优光谱指数是叶片水 分含量的重要指示指标(Yi et al., 2013)。因此, 采用 光谱反射率及其一阶或二阶微分值均可较好地指示 植被水分状况。

\section{2 光谱植被指数法}

光谱植被指数的建立在一定程度上考虑了植被 内部的物理机制, 具有一定物理含义。由于冠层光 谱是植被和环境背景的混合光谱, 用混合光谱与植 被参数建立相关关系必然受到较多随机因素的影响, 一个有效光谱指数必须能够将植被信息与环境背景 信息分离。植被光谱指数由于其简单又具有较好的 推广性, 广泛地用以获取植被生物物理特征属性。目 前, 已有多种方法考虑了植被含水量与光谱指数的 关系。基于400-2 $500 \mathrm{~nm}$ 光谱范围内对植被水分敏 感的波段有400、610、700、810、960、1240、1450、 1950、2 $500 \mathrm{~nm}$, 研究者们发展了比较成熟而又可 以用来监测植被水分的植被指数, 包括归一化差异 植被指数 $(N D V I) 、 W I$ 和归一化差异水分指数 $(N D W I)$ (Gao, 1996), 结合当前高光谱遥感技术的发展, $N D V I$ 和 $N D W I$ 监测植被水分的应用前景更为广泛 (张佳华等, 2007)。此外, 光合叶绿素苂光指数 (PRI) 也有着较好的应用。研究表明, NDWI适合估算单位 叶面积的水分含量, 而不适合估算单位植被干质量 的水分含量(Maki et al., 2004)。田永超等(2004)提出 了一种预测小麦水分状况的新的植被水分指数 $S R(610,560) / N D(810,610)(S R$, 比值植被指数; $N D$, 归一化植被指数)。沈艳等(2005)提出利用近红外和 短波红外波段反射率构造土壤可调节水分指数能有 效剔除土壤背景对冠层尺度叶片含水量提取的影
响 。 $Y i$ 等 (2013) 研究认为 $D R_{1647} / D R_{1133}$ 和 $D R_{1653} /$ $D R_{1687}(D R$, 一阶微分反射率值) 分别是 $E W T$ 和 $L F M C$ 最优估算指数, 以及 $Y i$ 等 $(2014)$ 提出新遥感 植被指数 $S R_{1060}\left(R_{1640} / R_{1060}\right)$ 和 $S R_{1640}\left(R_{1060} / R_{1640}\right)(S R$, 比值植被指数; $R$, 光谱反射率值) 与 $E W T$ 和 $C W C$ 显 著相关。Zhang和Zhou (2015)的研究表明, 绿光叶绿 素指数 $\left(C I_{\text {green }}\right)$ 、红边叶绿素指数 $\left(C I_{\text {red edge }}\right)$ 和红边归 一化比值指数 ( $N R_{\text {red edge }}$ 对 $C W C$ 和 $E W T$ 变异的响应 最为敏感, 建立了宽幅变异梯度下玉米(Zea mays) $C W C$ 和 $E W T$ 的高光谱遥感估算方法。该研究表明, 利用光谱植被指数对 $C W C$ 进行估算, $50 \%$ 光谱信息 来源于叶面积指数, 而且研究也提出, 在叶片/冠层 含水量高光谱遥感反演中, 如何看待植被结构光谱 信息与植被水分光谱信息二者之间的关系, 是抵消 还是促进? 如果是抵消, 那么如何有效地进行生长 信息与水分信息的分离? 这些问题值得进一步深入 探讨。

\section{3 二维特征空间统计回归模型法}

王鹏新等(2003)和韩丽娟等(2005)利用 NDVI与 地表温度 $\left(T_{\mathrm{s}}\right)$ 构成的特征空间进行了土壤水分和植 被覆盖状况的有效评价研究。该类方法通过监测植 被长势和地表辐射温度, 间接地反映植被和土壤含 水量, 是一种基于经验参数化的方法 (吴代晖等, 2010)。Price (1990)、Carlson等(1995)、Gillies 和 Carlson (1997)以及Sandholt等(2002)发现当研究区域 植被覆盖和土壤湿度变化范围较大时, 从遥感资料 获得的 $N D V I$ 和 $T_{\mathrm{s}}$ 所构成的散点图呈三角形, 并利用 土壤-植被-大气传输模型(SVAT)进行了验证。Moran 等(1994)从理论角度分析, 认为 NDVI- $T_{\mathrm{s}}$ 之间呈梯形 关系, 三角形空间成立的前提是观测数据包括了裸 露土壤, 长势极其良好的绿色植被, 以及长势一般 但特别枯黄的植被, 否则, 三角形的空间描述是不 成立的。Goward和Hope (1989)以及Price (1990)提出 了 $T_{\mathrm{s}}-N D V I$ 特征空间, Ridd (1995)、Gillies和Carlson (1995)、王鹏新等(2003) 和韩丽娟等 (2005) 利用 $T_{\mathrm{s}}-N D V I$ 特征空间进行了地面蒸散量分析及土壤水 分监测。围绕土壤水分遥感监测以可见光-近红外为 主要手段, 提出了不少模型与方法进行干旱监测 (詹志明等, 2006; 阿布都瓦斯提・吾拉木等, 2007), 而实际上, $T_{\mathrm{s}}$ 和反照率(albedo)的遥感反演既复杂又 包含了一定的误差, 进一步放大了土壤水分估算的 不确定性, 如何既简单又准确地监测植被或土壤水 
分状态是高光谱遥感反演领域的重要研究课题。值 得一提的是, 此类利用二维特征空间统计回归模型 法开展的研究, 往往既包括高植被盖度、中植被盖 度, 也包括低植被盖度的植被条件, 甚至裸露土壤 数据资料, 那么它将能够为有效探究多植被覆盖度 条件下植被含水量高光谱遥感反演以及植被含水 量普适性高光谱遥感估算模型构建提供新的研究 思路。

\section{4 基于光谱分析的全波段分析法}

一元/多元回归技术被广泛用来估算植被参数 和作物产量, 然而利用这些方法比如多元线性回 归、逐步回归估算植被生物物理/生物化学特征参数 时, 容易产生多重共线性问题(Curran, 1989; Mirzaie et al., 2014)。研究表明, 偏最小二乘回归分析 (PLSR)、人工神经网络(ANN)和主成分回归分析 (PCR) 在一定程度上能够减小多重共线性问题的影 响(Atzberger et al., 2010; Mirzaie et al., 2014)。此外, Mirzaie等(2014)研究揭示出偏最小二乘回归分析对 植被含水量的反演精度最高, 主成分回归分析对植 被含水量的反演精度最低, 原因在于主成分分析主 要侧重于对自变量的解释或归纳, 而偏最小二乘回 归分析则增强了自变量与因变量之间的相关关系。

\section{5 辐射传输模型法}

利用辐射传输模型进行叶片/冠层含水量的遥 感估算已有较多研究(Darvishzadeh et al., 2011)。理 论上认为冠层反射率变异是冠层、叶片和土壤背景 特征的函数, 存在一定的物理机理。目前广泛使用 的是PROSAIL辐射传输模型, 它是由SAILH冠层反 射率模型(Verhoef, 1984)和PROSPECT叶片光学特 征模型 (Jacquemoud \& Baret, 1990)结合, PROSPECT 是叶片尺度的模型拟合, 而SAILH是冠层尺 度光谱反射率拟合(Yi et al., 2014)。叶片尺度, PROSAIL使用叶片叶绿素含量 $\left(C_{\mathrm{ab}}\right)$, 叶片等量水厚 度 $(E W T)$ 、叶片结构参数 $(N)$ 和叶片干物质 $\left(C_{\mathrm{m}}\right)$ 作为 输入变量; 冠层尺度, 输入参数为 $L A I$ 、叶倾角分布 (leaf inclination angle distribution)、土壤亮度(soil brightness)、散射/直射比(ratio diffuse/direct irradiation)、太阳天顶角(solar zenith angle)、观测天顶角 (view zenith angle)和方位角(sun-view azimuth angle), 以及参数热点效应(hot-spot effect) (Clevers et al., 2010)。Yi等(2014)利用PROSPECT-5耦合SAILH研 究了与水分相关的遥感植被指数对 $E W T$ 和 $C W C$ 的
估算能力, 取得满意的研究结果。

总体而言, 辐射传输模型虽然具有一定理论基 础, 但不可避免地具有模型反演的缺陷(Darvishzadeh et al., 2011; Atzberger et al., 2015), 模型运行 所需要的特定参数(比如平均叶角、土壤背景反射 率), 尤其在复杂冠层条件下, 往往不易获取。在统 计学方法上, 不仅需要建立在大量数据观测以及所 获取的数据属性(数量、质量以及数据代表性), 而且 依赖于特定的传感器、研究位点和取样条件, 但该 方法易于开展(Atzberger et al., 2015), 一直沿用至 今, 并有广泛的应用。全波段分析方法在一定程度 上克服了多元线性回归、逐步回归的多重共线性问 题, 对于植被生物物理参数具有较好的预测能力 (Atzberger et al., 2010), 但难以克服过拟合问题(Ren \& Zhou, 2014), 而且Mirzaie等(2014)利用遥感植被 指数的任意波段组合进行植被含水量遥感估算时也 取得优于全波段分析方法的研究结果。综上所述, 各种研究方法各有千秋, 各有侧重, 各有优势, 虽 然不乏改进空间, 但它们均在植被含水量高光谱遥 感反演的发展进程中发挥着不可或缺的重要作用。

\section{4 问题和展望}

尽管植被含水量高光谱遥感监测已经取得了诸 多进展, 但植被含水量高光谱遥感监测的方法与指 标仍不统一, 严重制约着植被干旱胁迫的准确监 测、预警与评估。为此, 未来应重点强化以下方面 的研究:

(1)植被水分遥感指标及其对植被水分状况的 表征能力研究。虽然利用光学高光谱遥感技术有效 地开展了植被水分状况以及植被干旱胁迫的实时监 测与准确估算研究, 然而诸多植被水分指标中究 竟哪一个水分指标既适合于指示植被干旱胁迫状 态, 又易于接受高光谱遥感监测, 目前尚缺乏统一 的认识。

(2)环境背景对植被光谱的影响及其定量表达 研究。尽管光谱植被指数在一定程度上能够将植被 信息与环境背景信息分离, 然而稀疏植被条件下, 作物生长发育初期或干旱半干旱区, 冠层光谱受土 壤背景的强烈影响, 如何有效地分离土壤背景对植 被光谱的影响, 进而提高稀疏植被条件下植被含水 量高光谱遥感估算精度的问题函待解决。

(3)植被光谱中植物生长信息对水分信息的影 
响及其分离技术研究。在植被含水量高光谱遥感反 演中，面对植物生长、结构信息对水分信息的混淆、 干扰，如何有效地分离或减少植物生长对水分信息 遥感反演的影响, 对于利用遥感技术进行植被含水 量动态变化的实时有效监测意义重大。

(4)植被含水量多时空尺度监测研究。虽然已有 不少研究探讨了基于叶片、冠层、生态系统植被水 分状况与遥感信息之间的关系, 但不同尺度水分含 量与遥感信息之间关系的外推、内延或者对植被光 谱的贡献却鲜有报道, 且多尺度水分含量遥感监测 之间还缺乏明确的定量关系。

(5)植被干旱胁迫的遥感识别及其等级划分。植 被干旱胁迫程度因不同物种、同一物种不同发育阶 段而具有不同的阈值, 只有阐明植被含水量与植被 干旱胁迫状态之间的关系, 才能真正通过植被含水 量的遥感定量估算, 实现利用光学高光谱遥感技术 对植被干旱胁迫状态的实时监测预警。

因此, 未来研究拟从叶片、冠层和生态系统多 尺度协同开展植被含水量遥感监测研究, 重点解决 以下关键科学问题: 1)稀疏植被条件下植被含水量 高光谱遥感估算方法的建立; 2)适应于不同植被覆 盖条件下植被含水量普适性高光谱遥感估算模型的 发展; 3)植被含水量与植被干旱胁迫状态之间关系 的阐释; 以及4)区域尺度植被含水量遥感监测模型 的构建。通过多时空尺度植被含水量高光谱遥感估 算研究, 实现区域尺度植被干旱胁迫状态的遥感监 测与定量评估, 将为利用遥感技术进行大面积、无 破坏、实时评价植被生长状况, 以及植被干旱过程 及其产量的遥感监测与评估提供重要的科学依据。

致谢 感谢中国科学院植物研究所许振柱研究员在 工作中给予的指导与帮助。

\section{参考文献}

Atzberger C, Guérif M, Baret F, Werner W (2010). Comparative analysis of three chemometric techniques for the spectroradiometric assessment of canopy chlorophyll content in winter wheat. Computers and Electronics in Agriculture, 73, 165-173.

Atzberger C, Darvishzadeh R, Immitzer M, Schlerf M, Skidmore A, Le Maire G (2015). Comparative analysis of different retrieval methods for mapping grassland leaf area index using airborne imaging spectroscopy. International Journal of Applied Earth Observation and Geoinformation, 43, 19-31.
Barrs HD, Weatherley PE (1962). A re-examination of the relative turgidity technique for estimating water deficits in leaves. Australian Journal of Biological Sciences, 15, 413-428.

Bowyer P, Danson FM (2004). Sensitivity of spectral reflectance to variation in live fuel moisture content at leaf and canopy level. Remote Sensing of Environment, 92, 297-308.

Burgan RE (1996). Use of remotely sensed data for fire danger estimation. EARSeL Advances in Remote Sensing, 4(4), 1-8.

Cao Z, Wang Q, Zheng C (2015). Best hyperspectral indices for tracing leaf water status as determined from leaf dehydration experiments. Ecological Indicators, 54, 96-107.

Carlson TN, Capehart WJ, Gillies RR (1995). A new look at the simplified method for remote sensing of daily evapotranspiration. Remote Sensing of Environment, 54, 161- 167.

Ceccato P, Gobron N, Flasse S, Pinty B, Tarantola S (2002). Designing a spectral index to estimate vegetation water content from remote sensing data: Part 1: Theoretical approach. Remote Sensing of Environment, 82, 188-197.

Cho MA, Skidmore A, Corsi F, Wieren SE, Sobhan I (2007). Estimation of green grass/herb biomass from airborne hyperspectral imagery using spectral indices and partial least squares regression. International Journal of Applied Earth Observation and Geoinformation, 9, 414-424.

Chuvieco E, Rianõ D, Aguado I, Cocero D (2002). Estimation of fuel moisture content from multi temporal analysis of Landsat Thematic Mapper reflectance data: Applications in fire danger assessment. International Journal of Remote Sensing, 23, 2145-2162.

Clevers JGPW, Kooistra L, Schaepman ME (2008). Using spectral information from the NIR water absorption features for the retrieval of canopy water content. International Journal of Applied Earth Observation and Geoinformation, 10, 388-397.

Clevers JGPW, Kooistra L, Schaepman ME (2010). Estimating canopy water content using hyperspectral remote sensing data. International Journal of Applied Earth Observation and Geoinformation, 12, 119-125.

Cohen W, Maiersperger T, Gower S, Turner D (2003). An improved strategy for regression of biophysical variables and Landsat ETM+ data. Remote Sensing of Environment, 84, 561-571.

Colombo R, Meroni M, Marchesi A, Busetto L, Rossini M, Giardino C, Panigada C (2008). Estimation of leaf and canopy water content in poplar plantations by means of hyperspectral indices and inverse modeling. Remote Sensing of Environment, 112, 1820-1834.

Curran P (1989). Remote sensing of foliar chemistry. Remote Sensing of Environment, 30, 271-278.

Curran P, Dungan J, Peterson D (2001). Estimating the foliar biochemical concentration of leaves with reflectance spectrometry: Testing the Kokaly and Clark methodologies. Remote Sensing of Environment, 76, 349-359.

Danson FM, Steven MD, Malthus TJ, Clark JA (1992). High

www.plant-ecology.com 
spectral resolution data for determining leaf water content. International Journal of Remote Sensing, 13, 461-470.

de Jong SM, Addink EA, Hoogenboom P, Nijland W (2012). The spectral response of Buxus sempervirens to different types of environmental stres-A laboratory experiment. ISPRS Journal of Photogrammetry \& Remote Sensing, 74, 56-65.

de Jong SM, Addink EA, Doelman JC (2014). Detecting leafwater content in Mediterranean trees using high-resolution spectrometry. International Journal of Applied Earth Observation and Geoinformation, 27, 128-136.

Darvishzadeh R, Skidmore A, Atzberger C, Wieren SV (2008a). Estimation of vegetation LAI from hyperspectral reflectance data: Effects of soil type and plant architecture. International Journal of Applied Earth Observation and Geoinformation, 10, 358-373.

Darvishzadeh R, Skidmore A, Schlerf M, Atzberger C, Corsi F, Cho M (2008b). LAI and chlorophyll estimation for a heterogeneous grassland using hyperspectral measurements. ISPRS Journal of Photogrammetry \& Remote Sensing, 63, 409-426.

Darvishzadeh R, Atzberger C, Skidmore A, Schlerf M (2011). Mapping grassland leaf area index with airborne hyperspectral imagery: A comparison study of statistical approaches and inversion of radiative transfer models. ISPRS Journal of Photogrammetry \& Remote Sensing, 66, 894-906.

Deng B, Yang WN, Mu N, Zhang C (2016). The research of vegetation water content based on spectrum analysis and angle slope index. Spectroscopy and Spectral Analysis, 36, 2546-2552. [邓兵, 杨武年, 慕楠, 张超 (2016). 基于光 谱分析与角度斜率指数的植被含水量研究. 光谱学与 光谱分析, 36, 2546-2552.]

Dzikiti S, Verreynne JS, Stuckens J, Strever A, Verstraeten WW, Swennen R, Coppin P (2010). Determining the water status of Satsuma mandarin trees (Citrus unshiu Marcovitch) using spectral indices and by combining hyperspectral and physiological data. Agricultural and Forest Meteorology, 150, 369-379.

Gao BC (1996). NDWI-A normalized difference water index for remote sensing of vegetation liquid water from space. Remote Sensing of Environment, 58, 257-266.

Gillies RR, Carlson TN (1995). Thermal remote sensing of surface soil water content with partial vegetation cover for incorporation into climate models. Journal of Applied Meteorology, 34, 745-756.

Gillies RR, Carlson TN (1997). A verification of the 'triangle' method for obtaining surface soil water content and energy fluxes from remote measurements of the normalized difference vegetation index (NDVI) and surface radiant temperature. International Journal of Remote Sensing, 18, 3145-3166.

Goward SN, Hope AS (1989). Evaporation from combined reflected solar and emitted terrestrial radiation: Prelimi- nary FIFE results from AVHRR data. Advances in Space Research, 9, 239-249.

Ghulam A, Li ZL, Qin QM, Tong QX, Wang JH, Kasimu A, Zhu L (2007). A method for canopy water content estimation for highly vegetated surfaces-shortwave infrared perpendicular water stress index. Science in China Series D: Earth Sciences, 37, 957-965. [阿布都瓦斯提・吾拉木, 李 召良, 秦其明, 童庆禧, 王纪华, 阿里木江・卡斯木, 朱 琳 (2007). 全覆盖植被冠层水分遥感监测的一种方法: 短波红外垂直失水指数. 中国科学 $\mathrm{D}$ 辑: 地球科学, 37, 957-965.]

Han LJ, Wang PX, Wang JD, Liu SM (2005). Study on vegetation indices-surface temperature characteristic space. Science in China Series D: Earth Sciences, 35, 371-377. [韩 丽娟, 王鹏新, 王锦地, 刘绍民 (2005). 植被指数-地表 温度构成的特征空间研究. 中国科学 $\mathrm{D}$ 辑: 地球科学, 35, 371-377.]

Hunt Jr ER, Rock BN, Nobel PS (1987). Measurement of leaf relative water content by infrared reflectance. Remote Sensing of Environment, 22, 429-435.

Hunt Jr ER, Rock BN (1989). Detection of changes in leaf water content using near and middle-infrared reflectances. Remote Sensing of Environment, 30, 43-54.

Inoue Y, Morinaga S, Shibayama M (1993). Non-destructive estimation of water status of intact crop leaves based on spectral reflectance measurements. Japanese Journal of Crop Science, 62, 462-469.

Jackson RD (1985). Spectral response of cotton to suddenly induced water stress. International Journal of Remote Sensing, 6, 177-185.

Jacquemoud S, Baret F (1990). Prospect-A model of leaf optical properties spectra. Remote Sensing of Environment, 34, 75-91.

Jacquemoud S, Ustin S, Verdebout J, Schmuck G, Andreoli G, Hosgood B (1996). Estimating leaf biochemistry using the PROSPECT leaf optical properties model. Remote Sensing of Environment, 56, 194-202.

Kramer PJ (1983). Water Relations of Plants. New York Press, New York.

Kriedemamn PE, Barrs HD (1983). Photosynthetic Adaptation to Water Stress and Implication for Drought Resistance. Westbiew Press, Boulder.

Li BZ, Zhou GS (2014). Advance in the study on drought index. Acta Ecologica Sinica, 34, 1043-1052. [李柏贞, 周广胜 (2014). 干旱指标研究进展. 生态学报, 34, 1043- 1052.]

Liu C, Sun PS, Liu SR (2016). A review of plant spectral reflectance response to water physiological changes. Chinese Journal of Plant Ecology, 40, 80-91. [刘畅, 孙鹏森, 刘 世荣 (2016). 植物反射光谱对水分生理变化响应的研 究进展. 植物生态学报, 40, 80-91.]

Maki M, Ishiahra M, Tamura M (2004). Estimation of leaf water status to monitor the risk of forest fires by using remotely sensed data. Remote Sensing of Environment, 90, 441-450. 
Michio S, Tsuyoshi A (1989). Seasonal visible, near-infrared and mid-infrared spectral of rice canopies in relation to LAI and aboveground dry phytomass. Remote Sensing of Environment, 27, 119-127.

Mirzaie M, Darvishzadeh R, Shakiba A, Matkan AA, Atzberger C, Skidmore A (2014). Comparative analysis of different uni- and multi-variate methods for estimation of vegetation water content using hyper-spectral measurements. International Journal of Applied Earth Observation and Geoinformation, 26, 1-11.

Moran MS, Clarke TR, Inoue Y, Vidal A (1994). Estimating crop water deficit using the relation between surface-air temperature and spectral vegetation index. Remote Sensing of Environment, 49, 246-263.

Peñuelas J, Filella I, Biel C, Serrano L, Save R (1993). The reflectance at the 950-970 $\mathrm{nm}$ region as an indicator of plant water status. International Journal of Remote Sensing, 14, 1887-1905.

Peñuelas J, Filella I, Sweeano L (1996). Cell wall elastivity and Water Index (R970 nm/R900 nm) in wheat under different nitrogen availabilities. International Journal of Remote Sensing, 7, 373-382.

Price JC (1990). Using spatial context in satellite data to infer regional scale evaportranspiration. IEEE Transactions on Geoscience and Remote Sensing, 28, 940-948.

Qi Y, Dennison PE, Jolly WM, Kropp RC, Brewer SC (2014). Spectroscopic analysis of seasonal changes in live fuel moisture content and leaf dry mass. Remote Sensing of Environment, 150, 198-206.

Ren HR, Zhou GS, Zhang F, Zhang XS (2012). Evaluating cellulose absorption index (CAI) for non-photosynthetic biomass estimation in the desert steppe of Inner Mongolia. Chinese Science Bulletin, 57, 839-845. [任鸿瑞, 周广胜, 张峰, 张新时 (2012). 基于纤维素吸收指数(CAI)的内 蒙古荒漠草原非绿色生物量估算. 科学通报, 57, 839-845.]

Ren H, Zhou G (2014). Estimating aboveground green biomass in desert steppe using band depth indices. Biosystems Engineering, 127, 67-78.

Ridd MK (1995). Exploring a V-I-S (vegetation-impervious surface-soil) model for urban ecosystem analysis through remote sensing: Comparative anatomy for citied. International Journal of Remote Sensing, 16, 2165-2185.

Rollin EM, Milton EJ (1998). Processing of high spectral resolution reflectance data for the retrieval of canopy water content information. Remote Sensing of Environment, 65, 86-92.

Running SW, Gower ST (1991). Forest-BGC, a general model of forest ecosystem processes for regional applications II. Dynamic carbon allocation and nitrogen budgets. Tree Physiology, 9, 147-160.

Running SW, Nemani RR (1991). Regional hydrologic and carbon balance responses of forests resulting from potential climate change. Climatic Change, 19, 349-368.
Sandholt I, Rasmussen K, Andersen J (2002). A simple interpretation of the surface temperature/vegetation index space for assessment of surface moisture status. Remote Sensing of Environment, 79, 213-224.

Shen Y, Niu Z, Yan CY (2005). Estimation models for vegetation water content at both leaf and canopy levels. Chinese Journal of Applied Ecology, 16, 1218-1223. [沈艳, 牛铮, 颜春燕 (2005). 植被叶片及冠层层次含水量估算模型 的建立. 应用生态学报, 16, 1218-1223.]

Tang YL, Huang JF (2001). Study on hyperspectral remote sensing in agriculture. Remote Sensing Technology and Application, 16, 248-251. [唐延林，黄敬峰 (2001). 农业 高光谱遥感研究的现状与发展趋势. 遥感技术与应用, 16, 248-251.]

Tanner CB (1963). Plant temperature. Agronomy Journal, 50, 210-211.

Tian QJ, Gong P, Zhao CJ, Guo XW (2000). The feasibility of diagnosing wheat water status by using spectral reflectance. Chinese Science Bulletin, 45, 2645-2650. [田庆久, 宫鹏, 赵春江, 郭晓维 (2000). 用光谱反射率诊断小麦 水分状况的可行性. 科学通报, 45, 2645-2650.]

Tian YC, Zhu Y, Cao WX, Dai TB (2004). Relationship between canopy reflectance and plant water status of wheat. Chinese Journal of Applied Ecology, 15, 2072-2076. [田永 超, 朱艳, 曹卫星, 戴廷波 (2004). 小麦冠层反射光谱与 植株水分状况的关系. 应用生态学报, 15, 2072-2076.]

Ustin SL, Roberts DA, Gamon JA, Asner GP, Green RO (2004). Using imaging spectroscopy to study ecosystem processes and properties. BioScience, 54, 523-534.

Verhoef W (1984). Light scattering by leaf layers with application to canopy reflectance modeling: The SAIL model. Remote Sensing of Environment, 16, 125-141.

Wang JH, Zhao CJ, Guo XW, Huan WJ, Tian QJ (2000). Study on the water content of wheat leaves by the remote sensing. Acta Agriculturae Boreali-Sinica, 15(4), 68-72. [王纪华, 赵春江, 郭晓维, 黄文江, 田庆久 (2000). 利用遥感方法 诊断小麦叶片含水量的研究. 华北农学报, 15(4), 68-72.]

Wang JH, Zhao CJ, Guo XW, Tian QJ (2001). Study on the water status of the wheat leaves diagnosed by the spectral reflectance. Scientia Agricultura Sinica, 34, 104-107. [王纪 华, 赵春江, 郭晓维, 田庆久 (2001). 用光谱反射率诊断 小麦叶片水分状况的研究. 中国农业科学, 34, 104-107.]

Wang PX, Wan ZM, Gong JY, Li XW, Wang JD (2003). Advances in drought monitoring by using remotely sensed normalized difference vegetation index and land surface temperature products. Advance in Earth Sciences, 18, 527-533. [王鹏新, Wan ZM, 龚健雅, 李小文, 王锦地 (2003). 基于植被指数和土地表面温度的干旱监测模型. 地球科学进展, 18, 527-533.]

Wu DH, Fan WJ, Cui YK, Yan BY, Xu XR (2010). Review of monitoring soil water content using hyperspectral remote sensing. Spectroscopy and Spectral Analysis, 30, 30673071. [吴代晖, 范闻捷, 崔要奎, 间彬彦, 徐希孺 
(2010). 高光谱遥感监测土壤含水量研究进展. 光谱学 与光谱分析, 30, 3067-3071.]

Yebra M, Dennison PE, Chuvieco E, Riaño D, Zylstra P, Hunt Jr ER, Danson FM, Qi Y, Jurdao S (2013). A global review of remote sensing of live fuel moisture content for fire danger assessment: Moving towards operational products. Remote Sensing of Environment, 136, 455-468.

Yi Q, Bao A, Wang Q, Zhao J (2013). Estimation of leaf water content in cotton by means of hyperspectral indices. Computers and Electronics in Agriculture, 90, 144-151.

Yi Q, Wang F, Bao A, Jiapaer G (2014). Leaf and canopy water content estimation in cotton using hyperspectral indices and radiative transfer models. International Journal of Applied Earth Observation and Geoinformation, 33, 67-75.

Yilmaz MT, Hunt Jr ER, Goins LD, Ustin SL, Vanderbilt VC, Jackson TJ (2008). Vegetation water content during SMEX04 from ground data and Landsat 5 Thematic Mapper imagery. Remote Sensing of Environment, 112, 350-362.

Yuan WP, Zhou GS (2004). Theoretical study and research prospect on drought indices. Advances in Earth Science, 19, 982-991. [袁文平, 周广胜 (2004). 干旱指标的理论 分析与研究展望. 地球科学进展, 19, 982-991.]

Zhan ZM, Qin QM, Ghulam A, Wang DD (2006). A new method monitoring soil moisture based on NIR-Red spectral space. Science in China Series D: Earth Sciences, 36, 1020-1026. [詹志明, 秦其明, 阿布都瓦斯提・吾拉木, 汪冬冬 (2006). 基于NIR-Red光谱特征空间的土壤水分 监测新方法. 中国科学D辑: 地球科学, 36, 1020-1026.]

Zhang JH, Guo WJ, Yao FM (2007). The study on vegetation water content estimating model based on remote sensing technique. Journal of Basic Science and Engineering, 15, 45-53. [张佳华, 郭文娟, 姚凤梅 (2007). 植被水分遥感 监测模型的研究. 应用基础与工程科学学报, 15, 45-53.]

Zhang JH, Xu Y, Yao FM, Wang PJ, Guo WJ, Li L, Yang LM (2010). Advances in estimation methods of vegetation water content based on optical remote sensing techniques. Science China Technological Sciences, 53, 1159-1167.

Zhang JH, Li L, Yao FM (2010). Progress in retrieving vegetation water content under different vegetation coverage condition based on remote sensing spectral information. Spectroscopy and Spectral Analysis, 30, 1638-1642. [张佳 华, 李莉, 姚凤梅 (2010). 遥感光谱信息提取不同覆盖 下植被水分信号的研究进展. 光谱学与光谱分析, 30 , 1638-1642.]

Zhang F, Zhou GS (2014). Estimating canopy photosynthetic parameters in maize field based on multi-spectral remote sensing. Chinese Journal of Plant Ecology, 38, 710-719. [张峰, 周广胜 (2014). 玉米农田冠层光合参数的多光 谱遥感反演. 植物生态学报, 38, 710-719.]

Zhang F, John R, Zhou G, Shao C, Chen J (2014). Estimating canopy characteristics of Inner Mongolia’s grasslands from field spectrometry. Remote Sensing, 6, 2239-2254.

Zhang F, Zhou G (2015). Estimation of canopy water content by means of hyperspectral indices based on drought stress gradient experiments of maize in the North Plain China. Remote Sensing, 7, 15203-15223.

Zhang F, Zhou G, Christer N (2015). Remote estimation of the fraction of absorbed photosynthetically active radiation for a maize canopy in Northeast China. Journal of Plant Ecology, 8, 429-435.

Zhang F, Zhou G (2017). Deriving a light use efficiency estimation algorithm using in situ hyperspectral and eddy covariance measurements for a maize canopy in Northeast China. Ecology and Evolution, 7, 4735-4744.

Zhao Z, Li X, Yin YB, Tang J, Zhou SB (2010). Analysis of spectral features based on water content of desert vegetation. Spectroscopy and Spectral Analysis, 30, 2500-2503. [赵钊, 李霞, 尹业彪, 唐金, 周生斌 (2010). 荒漠植物 含水量的光谱特征分析. 光谱学与光谱分析, 30 , 2500-2503.]

Zhou S, Duursma RA, Medlyn BE, Kelly JWG, Prentice IC (2013). How should we model plant responses to drought? An analysis of stomatal and non-stomatal responses to water stress. Agricultural and Forest Meteorology, 182183, 204-214.

特邀编委: 黄 玫 责任编辑: 李 敏 